l'échauffement théorique, à cause de leur chaleur de fusion absorbant les calories.

Ne pas appliquer la méthode en pareil cas.

\section{e) Erreurs de Mesures}

10 Thermométriques. - II faut attendre que le thermomètre ail bien pris la température, ce qui, avec les thermomètres spéciaux employés donnant 2 à $8 \mathrm{c} / \mathrm{m}$ par degré, demande 1 minute $1 / 2$ environ ; donner de légers chocs à l'ongle pour s'assurer que la colonne de mercure a bien pris sa place.

Il faut aussi lire $\theta_{2}^{\prime}$ et $A_{2}$, avec le thermomètre vertical (il est étalonné ainsi), ou tout au moins incliné également dans les mesures (influence de la pression de la colonne de mercure dilalant plus ou moins le réservoir du thermomètre).

Il est commode de disposer d'un jeu de thermomètres courts et sensibles :

$$
\begin{aligned}
& \text { L'un gradué de } 0^{\circ} \text { à } \mathrm{T}_{1} \\
& \text { L'autre de } \mathrm{T}_{1}-2^{\circ} \text { à } \mathrm{T}_{2} \\
& \text { L'autre de } \mathrm{T}_{2}-2^{\circ} \text { à } \mathrm{T}_{3}
\end{aligned}
$$

et ainsi de suite.

Il faut vérifier qu'il n'y a pas d'interruption dans la colonne de mercure.

$2^{\circ}$ Manométriques. - Si l'on n'a pas confiance dans les mano mètres de l'usine, il est facile de brancher un manomètre étalon, exact à moins de $1 \%$ près.

On connaît bien généralement la hauteur de chute totale de l'usine, donc la pression statique nette que doit indiquer le manomètre quand il n'y a pas de débit, ce qui est un moyen de le vérifier.

La lecture de la pression manométrique a autant d'importance que celle des températures. Si l'on n'avait qu'un mano- mètre douteux il serait préférable de se baser sur la hauteur de chute réelle connue par nivellement à la conslruction de l'usine, et de calculer les pertes de charge dans la conduite forcée, pour le débit évalué approximativement.

Enfin, les mesures donnant le rendement étant très rapidts, on peut les recommencer plusieurs fois pour avoir une certitude plus grande sur les résultats.

f) Stabilisation des régimes. - Il est enfin utile de revenir sur la nécessité avant de faire la série de mesures thermométriques correspondant à un régime donné, d'attendre un temps suffisant pour la stabilisation des différentes températures des pièces el des parois. 5 à 10 minutes suffisent généralement pour cela, si les débits d'eau en jeu sont suffisamment forts par rapport à l'ambiance.

Telles sont les principales précautions indiquées par l'expérience déjà acquise.

En résumé, l'application correcte de la formule :

$$
\eta=\frac{427\left(\Theta_{2}^{\prime}-\Theta_{2}\right)}{H}
$$

nécessite :

- La mesure correcte de la température de détente $\Theta^{\prime}{ }_{Q}$ de l'eau affluant à la turbine.

- La mesure correcte de la température $\Theta_{9}$ de l'eau s'échappant au même instant de la turbine.

- La mesure correcte de la pression manométrique $\mathrm{H}$ existant à cet instant sur la turbine.

Le degré d'approximation sera celui résultant de ces trois mesures.

Il pourra souvent être à moins de $1 \%$ près.

\title{
Sur l'utilisation de très petites sources d'énergie hydraulique pour les besoins de l'Agriculture.
}

\author{
Par L. RIGOTARD, Iàgénieur Agronome à Chaàtesse (Isère)
}

Depuis un cértain temps on envisage de plus en plus l'emploi de l'énergie mécanique pour exécuter les travaux agricoles. C'est une application d'une incontestable utilité pour notre production nationale ; cependant la mise au point de cette adaptation de l'énergie hydraulique ou électrique apparaît ardue dans la pratique. On peut ici rappeler pour mémoire qu'il est assez difficile de fournir du courant électrique à des abonnés utilisant la force d'une façon très irrégulière et dans bien des cas pendant un très pelit nombre d'heures par an. D'autre part, il est gênant et onéreux, pour des raisons assez nombreuses de divers ordres, pour une grande ligne de transport d'énergie, d'effectuer' en cours de route des distributions aux petites agglomérations rurales, très nombreuses et de peu d'importance moyenne,

Il semble que l'on pourrait envisager l'utilisation sur une base très étendue des nombreux petits cours d'eau qui sillonnent nos campagnes, pour fournir sur place à ces populations rurales l'énergie que la rareté de main-d'œuvre leur fait une obligation d'utiliser.

Le grand obstacle à cette utilisation de la houille blanche des très petits cours d'eau réside dans le coût élevé des installations de faible puissance. Or les agriculteurs n'utilisant l'énergie qu'un très petit nombre d'heures par jour ou même, de plus, peu de jours par an, il importerait de pouvoir réaliser des installations dont les frais d'amortissement et d'entretien ne s'éloignent pas trop de l'ordre de grandeur des frais de main-d'œuvre économisés. Dans la plupart des cas il ne s'agirait pas de faire des installations ayant, au point de vue technique, une certaine perfection, mais d'utiliser à peu de frais une partie de l'énergie disponible.

Les agriculteurs ont d'ailleurs l'esprit ainsi fait que dès qu'ils ont reconnu les avantages d'une méthode, ils se lancent d'euxmêmes dans l'exagération de son emploi. Si donc on amène un certain nombre d'entre eux à faire des installations qui leur donnent satisfaction sous le rapport de la commodité comme sous le rapport financier, on peut être sûr de voir se développer rapidement l'utilisation de la houille blanche dans les campagnes. Il existe dans les communes rurales sur une partie importante du territoire de la France une quantité de houille blanche suffisante pour l'agriculture qui y est pratiquée. Ainsi dans un groupe de trois communes contiguës du département de l'Isère que nous prenons comme exemple, il existe, à proximité ou même enclavées dans des exploitations agricoles, un certain nombre de chutes d'eau aménageables. Répartie sur quatre ou cinq ruisseaux dont plusieurs débitent environ cent litres à la seconde et pouvant donner facilement des chutes de 20 à 30 mètres, la puissance 
disponible dépasse cinquante chevaux ; c'est-à-dire que poụr les communes envisagées, la puissance des cours d'eau qui arrosent leur sol est très supéricure au maximum que les populations rurales sont capables d'utiliser. Or il s'agil de localités où il n'exisle aucune distribution d'énergic éleclrique, où les frais de lignes d'amenée seraient élevés et hors de proportion avec le produit financier qu'une société pourrait en tirer. Il n'est pas douteux que de nombreuses communes rurales se trouvent dans des siluations comparables à celle-ci.

Il semble ruc daus les conditions actuelles les installations hydrauliques de faible puissance soient un peu onéreuses. Cela ne tiendrait-il pas à ce fait que les turbines sont calculées et construites pour chaque chute envisagée. Ne serait-il pas possible d'arriver à construire ces appareils en série. On serait amené à préparer non plus une turbine pour une chute déterminée, mais une chule pour une turbine dont les caractéristiques seraient convenablement encadrées par celles de la chute d'eau à utiliser.

Enfin il faut faire l'éducation et l'instruction pratique spéciale des agriculteurs intéressés, tant en ce qui concerne l'utilisation de la houille blanche qu'en ce qui concerne l'usage de l'énergie éleclrique. Pour atteindre ce but, la réalisation de quelques installations modèles pouvant servir à des démonstrations, l'équipement de wagons de chemins de fer, ou même de camions automobiles, pour en faire des centres de démonstration et d'apprentissage circulant dans les régions agricoles, paraissent des moyens efficaces à proposer. On peut donc souhaiter que, sous le haut patronage des services intéressés de l'Etat, cette propagande soit entreprise, d'une façon désintéressée au point de vue immédiat, par les constructeurs, avec l'aide des Compagnies de chemins de fer, qui ont obtenu déjà par leur puissante action dans d'autres domaines de l'organisation agricole de si remarquables résultats,

\title{
Les Conducteurs électriques d'aluminium et d'aluminium-acier.
}

\author{
Par M: DUSAUGEY, Ingénieur civil des Mines à Grenoble.
}

\section{HistoriQUe}

Bien cue l'aluminium ne possède pas en lui-même des propriétés mécaniques très remarquables, sa faible densité et sa conductibilité électrique relativement élevée ont depuis longtemps attiré l'altention des élcctriciens, surtout à partir du jour où son prix de revient lui a permis de compter au nombre des métaux usuels.

Il est assez difficile de savoir si c'est en Europe ou en Amérique que l'on a pour la première fois utilisé l'aluminium comme conducteur électrique. Ce qu'il y a de certain c'est qu'il y a bien une trentaine d'années que les premiers essais ont été effectués en France.

Ils ne donnèrenl pas des résultats bien séduisants. La qualité du métal était assez mauvaise, la nature et la proportion des impurelés nuisibles assez mal connues, leur influence sur les propriétés mécaniques et électriques du mélal complètement inconnue et enfin la technique d'emploi inexistante. On subit donc des mécomples qui, bien entendu, rendirent l'aluminium suspect pendant de nombreuses années, de même que le cuivre étail demeuré suspect pendant près de 30 ans.

Aujourd'hui, après 25 ans de perfectionnement dans la fabrication du métal et de ses alliages et dans les méthodes d'emploi des produits labriqués, l'emploi de l'aluminium en électricilé s'esl généralisé et l'aluminium est officiellement admis par les règlements administratifs de la plupart des nations, au même titre que le cuivre, dans la construction des installations électriques. Il ne sera donc bientôt plus nécessaire de consacrer à ce métal une attention plus particulière qu'aux autres matériaux de l'électrotechnique.

L'aluminium a fait l'objet, au point de vue scientifique el au point de vue technique, d'un grand nombre d'études qui ont été synthétisées dans une série de conférences organisées à Paris du 21 au 29 mai 1921, par la Société d'Encouragement pour l'Industrie Nationale: Ces conférences, faites à l'occasion d'une exposition publique des applications de l'aluminium, ont fait ressortir les progrès considérables réalisés en ces dernières années dans la métallurgie et dans l'emploi de ce métal. Tous les industriels qui sont appelés à bénéficier de ces progrès liront avec fruil calle série de conférences. Mais le plus importanl travail au point de vue des applications électricues, semble être celui qui a été exécuté, au cours des années 1918 et 1919. par l'Union des Syndicats de l'Electricité.

Dans un substantiel rapport, l'Union des Syndicats de l'Electricité montre le très grand intérêt économique qu'il y a, pour les pays pauvres en cuivre, à faire un large usage de l'aluminium. Elle décrit les résultats pratiques acquis à ce jour dans l'emploi de ce métal. Elle définit ses propriétés chimiques, mécaniques et. électriques lorsqu'il doit servir à la constitution des conducteurs électriques. Enfin elle fait ressortir ses avantages économiques sur le cuivre et elle indique les principes généraux à observer dans la construction des installations électriques.

Il est impossible de discuter aujourd'hui les emplois de l'aluminium en électricité sans se référer à cet important travail. C'est ce què nous ferons dans le présent rapport.

\section{COMPARAISON TEGHNIQUE ET ÉCONOMIQUE DE L'ALUMINIUM ET DU CUIVRE}

Après avoir rappelé que les mécomptes subis lors des premiers essais étaient dus à la composition chimique du métal et aux mauvaises méthodes d'emploi des produits fabriqués, l'Union des Syndicats de l'Electricité, guidée par les résultats de l'expé rience acquise à ce jour, définit les propriétés de l'aluminium industricl qui doit satisfaire aux exigences de l'industrie de l'électricité

Un métal trop pur n'aurait pas assez de ténacité, un métal trop impur n'aurait pas une conductibilité électrique suffisante. La dose optimum d'impuretés ( $\mathrm{Fe}, \mathrm{Si}$. C et $\mathrm{O}$ ) a été fixée à $1 \%$, élant cntendu que le sodium, facteur probable de l'altération du métal, ne serait jamais en quantité perceptible à l'analyse chimique. A cette dose d'impuretés, correspond une résistance mécanique, à l'état recuil, de 8,5 à $9 \mathrm{~kg}$ par $\mathrm{mm}^{2}$ et une résistivité de 2,89 microhms-cenlim. à $20^{\circ}$. Si le métal est écroui, la résistance mécanique peut atteindre, pour des fils de 1,5 à $2 \mathrm{~mm}$., c'est-à-dire fortement écrouis par le tréfilage, 20 à $22 \mathrm{~kg}$ par mm2 tandis que la résistivité s'accroit légèrement de $1 \%$ environ, et passe à 2,95 microhms-cent.

On trouve de suite dans ces chiffres et dans ceux d'un tableau comparatif très complet des propriétés mécaniques ęt électriques de l'aluminium commercial et du cuivre commercial (Ta- 